\title{
Things We Do For No Reason ${ }^{\mathrm{TM}}$ : Failing to Question a Penicillin Allergy History
}

\author{
Renee S Kleris, MD, MPH'1 , Patricia L Lugar, MD, MS ${ }^{1,2 *}$
}

'Department of Pediatrics, Duke University Medical Center. Durham, North Carolina; ${ }^{2}$ Department of Medicine, Duke University Medical Center, Durham, North Carolina.

Inspired by the ABIM Foundation's Choosing Wisely ${ }^{\circledR}$ campaign, the "Things We Do for No Reason TM" (TWDFNR) series reviews practices that have become common parts of hospital care but may provide little value to our patients. Practices reviewed in the TWDFNR series do not represent "black and white" conclusions or clinical practice standards but are meant as a starting place for research and active discussions among hospitalists and patients. We invite you to be part of that discussion.

\section{CLINICAL SCENARIO}

An 80-year-old male - with a past medical history significant for hypertension, atrial fibrillation, and type II diabetes mellituspresented to the hospital with fevers, confusion, and urinary outflow tract difficulties. On exam, he was noted to have mild suprapubic tenderness with flank tenderness. Blood and urine cultures grew Enterococcus faecalis sensitive to ampicillin. Because of the patient's listed penicillin (PCN) allergy, he was started on aztreonam and vancomycin instead of ampicillin.

\section{WHY YOU MIGHT SIMPLY ACCEPT A PCN ALLERGY HISTORY}

Ten percent of the United States population reports an allergy to penicillin and derivatives-one of the most commonly reported drug allergies. ${ }^{.}$Allergic reactions to drugs are distinct immune reactions mediated by drug-specific immunoglobulin E (IgE) that are potentially life-threatening. Specifically these allergic reactions are called IgE-mediated, type 1 hypersensitivity reactions which are characterized by hives; itching; flushing; tissue swelling, especially in areas of the face and neck; bronchospasm; and gastrointestinal (Gl) symptoms, including cramping and diarrhea. Head and neck swelling can quickly result in airway compromise. Profound fluid extravasation and release of mediators from mast cells and basophils can rapidly drop blood pressure. Anaphylaxis requires rapid intervention to prevent severe complications and death. Given the life-threatening consequences of anaphylaxis, a cautious approach before administering PCN to $\mathrm{PCN}$-allergic patients is mandatory.

\section{WHY YOU SHOULD QUESTION} \section{A REPORTED PCN ALLERGY}

While $10 \%$ of the adult population and $15 \%$ of hospitalized adults report PCN allergy, clinical studies suggest that $90 \%$ of all patients reporting a PCN allergy can tolerate PCN antibiotics. ${ }^{1-3}$ There are several reasons patients initially labeled as PCN allergic may later be able to tolerate this drug. First, patients can lose sensitivity to specific PCN IgE antibodies over time if PCN is avoided. ${ }^{4}$ Second, non-lgE-mediated immune reactions of skin or $\mathrm{Gl}$ tract are often wrongly attributed to an IgE-mediated process from a concurrent medication (Table). For example, viral infections can cause exanthems or hives which may be mistaken for an antibiotic-associated lgE-meditated allergic reaction. ${ }^{6}$ These non-lgE skin reactions include severe manifestations including Stevens-Johnson syndrome (SJS) and toxic epidermal necrolysis or benign adverse reactions such as Gl upset, dizziness, or diarrhea which are often misclassified as an allergy, and this error is perpetuated in the medical record. Third, patients may report a PCN allergy for themselves when a family member is possibly allergic.

PCN allergy has risen to the level of a public health issue as PCN-allergic patients are often relegated to second-line broad-spectrum antibiotics. ${ }^{7}$ This public health issue is exacerbated when patients with faux or resolved PCN allergy receive the same treatment. Patients labeled as PCN allergic-whether correctly or incorrectly-have poorer outcomes as noted by increased rates of serious infections and tend to have longer hospital stays. ${ }^{8-10}$ Treatment-related secondary infections from the use of broad-spectrum antibiotics, such as Clostridiodes difficile and vancomycin-resistant Enterococcus, are identified more frequently in PCN-allergic patients. ${ }^{7}$ Additionally, pregnant women with PCN allergy, with or without group B streptococcus infections, have higher rates of cesarean sections and longer hospitalizations. ${ }^{11}$ The misuse and overuse of antibiotics, especially broad-spectrum medications, has led to resistant bacteria that are increasingly difficult to treat. ${ }^{7}$ Treating with the most narrow-spectrum antibiotic whenever possible is critical. Overall, failure to address and assess PCN allergy can result in treatment failures and unnecessary broad-spectrum antibiotic use.

\section{WHEN YOU SHOULD BELIEVE A REPORTED PCN AND BETA-LACTAMS ALLERGY HISTORY}

Avoid beta-lactams for patients with a reported allergy who are medically frail (eg, critically ill intensive care unit patients and those unable to communicate) or have a documented allergic reaction to a beta-lactam within five years. An estimated $50 \%$ of patients who had a documented true IgE-mediated allergic reaction within five years of a documented true allergic reaction

*Corresponding Author: Patricia Lugar, MD, MS; E-mail: patricia.lugar@duke. edu; Telephone: 919-684-6122

Published online first March 20, 2019.

Received: June 8, 2018; Revised: December 21, 2018;

Accepted: January 19, 2019

(c) 2019 Society of Hospital Medicine DOI 10.12788/jhm.3170 
TABLE. Typical IgE-Mediated vs Non-IgE-Mediated Drug Reactions

\begin{tabular}{lcccc}
\hline & Common Symptoms & Cause and Mediators Involved & Timing & Example \\
\hline IgE-Mediated Reaction & Hives, swelling, shortness of breath & Histamine from mast cells & $\begin{array}{c}\text { Immediate (typically within } \\
15 \text { minutes to an hour) }\end{array}$ & $\begin{array}{c}\text { Patient develops hives } \\
15 \text { minutes after receiving amoxicillin }\end{array}$ \\
\hline Non-lgE-Mediated Reaction & $\begin{array}{c}\text { Delayed maculopapular rash or can } \\
\text { have severe findings including blisters, } \\
\text { mucosal membrane involvement, and } \\
\text { intestinal tract involvement (eg, Gl } \\
\text { symptoms such as diarrhea) }\end{array}$ & $\begin{array}{c}\text { T cell mediated and various other } \\
\text { mechanisms }\end{array}$ & $\begin{array}{c}\text { Delayed (days to weeks) } \\
\text { Maculopapular rash ( } 24 \text { hours) }\end{array}$ & $\begin{array}{c}\text { Patient develops a pruritic rash } \\
2 \text { weeks after starting a medication } \\
\text { that has since been stopped }\end{array}$ \\
AGEP (1-11 days) & SIS/TENS (4-28 days) \\
DRESS syndrome (2-6 weeks)
\end{tabular}

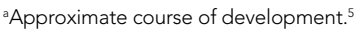

Abbreviations: AGEP, Acute generalized exanthematous pustulosis, DRESS syndrome, drug reaction with eosinophilia and systemic symptoms; GI, gastrointestinal; IgE, immunoglobulin E; SJS/ TENS; Stevens-Johnson syndrome/Toxic epidermal necrolysis.

remain allergic to $\mathrm{PCN}$ and are at risk for an allergic reaction with reexposure. ${ }^{1}$ PCN allergy evaluation with $\mathrm{PCN}$ skin testing (PST) and oral challenge in patients who had a reaction within five years have a higher risk of a fatal outcome with an oral challenge despite negative skin testing. PCN allergy evaluation is best handled on a case by case basis in this population.

\section{WHAT YOU SHOULD DO INSTEAD}

Obtain a thorough drug allergy history. If the history is not consistent with a personal history of an IgE-mediated reaction to PCN ever or if there is documentation that PCN was administered and tolerated since the reaction (eg, a dental prescription), a PCN or beta-lactam can be given. An exception to this rule are patients with a history of an allergic reaction to both a cephalosporin and a PCN-approach this as two separate allergies. Remove the PCN allergy if it is not consistent with the history of IgE-mediated reaction or the patient subsequently had tolerated a PCN/PCN derivative. Regarding the cephalosporin issue, patients are often allergic to a side chain of the cephalosporin and not to the beta-lactam ring. Patients should avoid the specific cephalosporin unless the history is also not consistent with an IgE-mediated reaction or the patient had subsequently tolerated this medication. An allergy evaluation can be useful to discern next steps for cephalosporin allergy. Once the antibiotic is administered and tolerated, the medical record should be updated as well to prevent future mislabeling.

If the symptoms associated with a reported history of a PCN allergy are unknown or consistent with an IgE-mediated reaction, or the patient has not been exposed to PCN since the allergic reaction, the patient should undergo PST followed by a supervised oral test dose to determine whether the allergy exists or persists. PCN allergy evaluation is a simple two-step process of PST followed by an oral challenge of amoxicillin. The use of PCN allergy testing as described is validated and safe. ${ }^{12}$ A negative skin prick and intradermal test have a negative predictive value that approaches $100 \%{ }^{12,13}$ Completing the final step-the oral challenge-eliminates concerns for false-negative testing results and patient fears. Additionally, once a patient has had negative skin testing and passed an oral challenge, he/she is not at increased risk of resensitization after PCN/PCN derivative use. ${ }^{14}$
Although the test takes one and a half hours on average, the benefits that follow are lifelong. Improving future management by disproving a reported allergy affects an individual patient's clinical course globally, results in cost savings, and increases the use of narrow-spectrum antimicrobials. It is particularly important to test $\mathrm{PCN}$-allergic patients preemptively who are at high risk of requiring PCN/PCN derivative antibiotics. High-risk patients include, but are not limited to, surgery, transplant, hematology/oncology, and immunosuppressed patients. Inpatients with PCN allergy have higher antibiotic costs-both for medications used during their hospitalization and also for discharge medications. ${ }^{15}$ A study by Macy and Contreras compared the cost of skin testing to money saved by shortening hospitalization days for 51,582 patients with PCN allergy. The cost for testing was $\$ 131.37$ each (total of $\$ 6.7$ million). The testing contributed to a $\$ 64$ million savings for the three-year study period_-savings that is 9.5 times larger than the cost of the evaluation. ${ }^{8}$ A smaller study that looked at cost-effectiveness of PST for 50 patients found an overall cost savings of $\$ 11,005$ due to the antimicrobial choice alone (\$297 per patient switched to a beta-lactam antibiotic). ${ }^{16}$

\section{RECOMMENDATIONS}

- Obtain a thorough drug allergy history as many "allergic reactions" can be removed by history alone. Update the medical record if you can confirm a patient has since tolerated PCN or a PCN derivative to which they were previously allergic. Offer a supervised oral challenge if the patient has any concerns.

- Perform PST if a patient has a PCN allergy listed in their chart and the allergy history is unclear. A negative skin test should be followed by a supervised oral challenge to PCN/PCN derivative if skin testing is negative.

- Test PCN-allergic patients preemptively who are at high risk of requiring PCN/PCN derivative antibiotics. High-risk patients include surgery, transplant, hematology/oncology, and immunosuppressed patients.

- Implement published protocols from allergists for healthcare systems that lack access to allergy physicians.

- Do not perform PST on patients with a history that is suggestive of a non-lgE-mediated allergic reaction. For these cases, patients are advised to avoid the medication. A supervised 


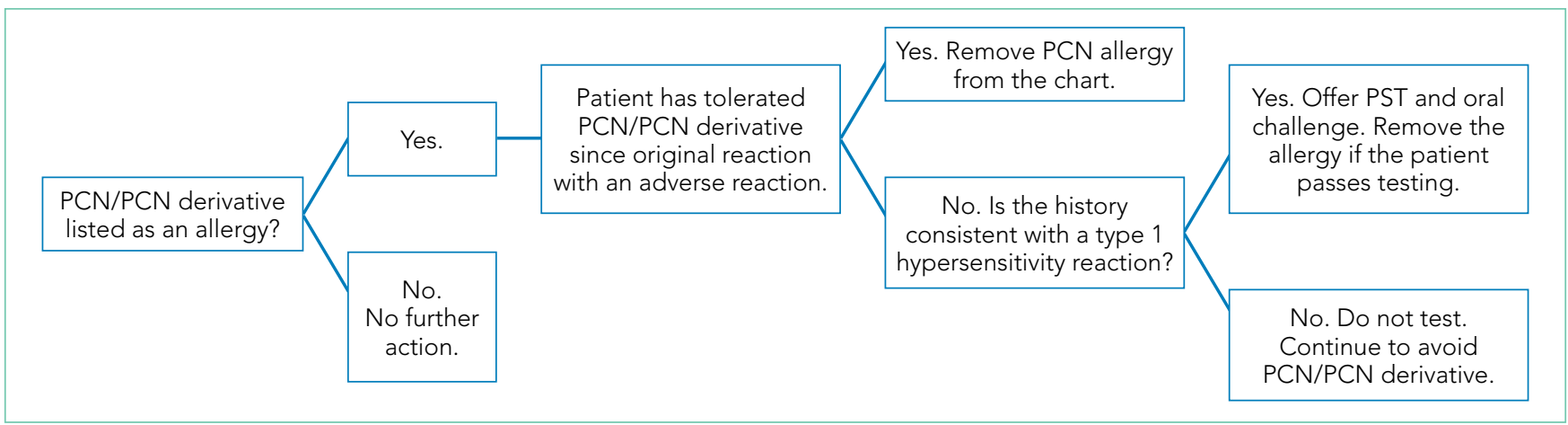

FIG. Penicillin Allergy Evaluation

graded oral challenge can be considered on a case-by-case basis if the reaction was not a severe cutaneous adverse reaction syndrome, like SJS, and the benefit of using the medication outweighs the potential harm.

\section{CONCLUSION}

The patient, in this case, reported an allergic reaction to PCN over 50 years before this presentation. The reported reaction immediately after receiving IV PCN was a rash-a symptom concerning for an IgE-mediated reaction. Since the patient is well over 10 years from his allergic reaction and would benefit from a PCN derivative, PST testing should be pursued.

The patient passed his skin testing and an oral challenge dose of amoxicillin. With the PCN allergy removed from his chart, his medical team transitioned him from aztreonam and vancomycin to ampicillin. He was then discharged home on amoxicillin and informed that he might be safely treated with $\mathrm{PCN} / \mathrm{PCN}$ derivatives in the future.

Given the rise in antimicrobial resistance and both the clinical implications and increased costs associated with PCN allergy, it is crucial to offer an allergy evaluation to patients identified as PCN allergic. Hospitalists play a crucial role in obtaining the initial history, determining if the patient has tolerated the antibiotic since the initial reaction, and identifying patients who may benefit from further evaluation for PCN allergy. In hospitals with PST available for inpatients, testing can be performed during the admission. Additionally, it is essential that allergists work with hospitalists and primary care physicians to provide seamless access to outpatient drug allergy evaluations (PST followed by oral challenge) to address the issue of PCN allergy before an acute need for a PCN/PCN derivative antibiotic in the hospital.

Do you think this is a low-value practice? Is this truly a "Thing We Do for No Reason "TM?" Share what you do in your practice and join in the conversation online by retweeting it on Twitter (\#TWDFNR) and liking it on Facebook. We invite you to propose ideas for other "Things We Do for No Reason TM" topics by e-mailing TWDFNR@hospitalmedicine.org.

Disclosures: The authors have no conflicts of interest

Funding: This work is supported by the following NIH Grant: T-32 Al007062-39.

\section{References}

1. American Academy of Allergy, Asthma and Immunology, the American College of Allergy, Asthma and Immunology, and the Joint Council of Allergy, Asthma and Immunology. Drug allergy: an updated practice parameter. Ann Allergy Asthma Immunol. 2010;105(4):259-273. https://doi.org/10.1016/j. anai.2010.08.002.

2. American Academy of Allergy Al. Ten things physicians and patients should question Choosing Wisely, ABIM Foundation 2014. http://www.choosingwisely.org/clinician-lists/american-academy-allergy-asthma-immunlogy-non-beta-lactam-antibiotics-penicillin-allergy/. Accessed October 23, 2017.

3. Blumenthal KG, Wickner PG, Hurwitz $S$, et al. Tackling inpatient penicillin allergies: Assessing tools for antimicrobial stewardship. J Allergy Clin Immunol. 2017;140(1):154-161. https://doi.org/10.1016/j.jaci.2017.02.005.

4. Blanca M, Torres MJ, Garcia JJ, et al. Natural evolution of skin test sensitivity in patients allergic to beta-lactam antibiotics. J Allergy Clin Immunol. 1999;103(5):918-924. https://doi.org/10.1016/S0091-6749(99)70439-2.

5. Duong TA Valeyrie-Allanore $L$, Wolkenstein $P, C$ Chosidow $O$. Severe cutaneous adverse reactions to drugs. Lancet. 2017;390(10106:1996-2011. doi:10.1016/ S0140-6736(16)30378-6.

6. Gonzalez-Estrada A, Radojicic C. Penicillin allergy: a practical guide for clinicians. Cleve Clin J Med. 2015;82(5):295-300. https://doi.org/10.3949/ ccjm.82a.14111.

7. Solensky R. Penicillin allergy as a public health measure. J Allergy Clin Immunol. 2014;133(3):797-798. https://doi.org/10.1016/j.jaci.2013.10.032.

8. Macy E, Contreras R. Health care use and serious infection prevalence associated with penicillin "allergy" in hospitalized patients: a cohort study. J Allergy Clin Immunol. 2014;133(3):790-796. https://doi.org/10.1016/j. jaci.2013.09.021

9. Chen JR, Khan DA. Evaluation of penicillin allergy in the hospitalized patient: opportunities for antimicrobial stewardship. Curr Allergy Asthma Rep. 2017;17(6):40. https://doi.org/10.1007/s11882-017-0706-1.

10. Blumenthal KG, Wickner PG, Hurwitz $S$, et al. Tackling inpatient penicillin allergies: Assessing tools for antimicrobial stewardship. J Allergy Clin Immunol. 2017;140(1):154-161. https://doi.org/10.1016/j.jaci.2017.02.005.

11. Desai SH, Kaplan MS, Chen Q, Macy EM. Morbidity in pregnant women associated with unverified penicillin allergies, antibiotic use, and group B Streptococcus infections. Perm J. 2017;21. https://doi.org/10.7812/TPP/16080 .

12. Macy E, Ngor EW. Safely diagnosing clinically significant penicillin allergy using only penicilloyl-poly-lysine, penicillin, and oral amoxicillin. J Allergy Clin Immunol Pract. 2013;1(3):258-263. https://doi.org/10.1016/j.jaip.2013.02.002.

13. Solensky R. The time for penicillin skin testing is here. J Allergy Clin Immunol Pract. 2013;1(3):264-265. https://doi.org/10.1016/j.jaip.2013.03.010.

14. Solensky R, Earl HS, Gruchalla RS. Lack of penicillin resensitization in patients with a history of penicillin allergy after receiving repeated penicillin courses. Arch Intern Med. 2002;162(7):822-826.

15. Sade K, Holtzer I, Levo Y, Kivity S. The economic burden of antibiotic treatment of penicillin-allergic patients in internal medicine wards of a general tertiary care hospital. Clin Exp Allergy. 2003;33(4):501-506. https://doi. org/10.1046/j.1365-2222.2003.01638.x.

16. King EA, Challa S, Curtin P, Bielory L. Penicillin skin testing in hospitalized patients with beta-lactam allergies: effect on antibiotic selection and cost. Ann Allergy Asthma Immunol. 2016;117(1):67-71. https://doi.org/10.1016/j. anai.2016.04.021. 